\title{
La importancia de las subjetividades para la generación de una educación transformadora en ambientes virtuales
}

\author{
Milena Alcócer Tocora
}

\begin{abstract}
Resumen
Las subjetividades se manifiestan como una posibilidad importante dentro de la educación en ambientes virtuales debido a que se pueden constituir como una herramienta que permite generar verdaderos aprendizajes; de acuerdo con lo anterior, el presente documento hace primero un recorrido sobre el concepto de subjetividad y las relaciones existentes entre los contextos cultural, tecnológico y educativo. De otro lado se presenta una reflexión que pretende profundizar sobre los alcances e implicaciones de las relaciones que pueden propiciarse entre las subjetividades, los procesos de formación y la educación virtual específicamente.
\end{abstract}

Palabras clave: Subjetividades, Ambientes virtuales, Educación.

1 Licenciada en Biología y Química, Especialista en Química de Productos Naturales, Magister en Docencia de la Química. Docente Ocasional Escuela de Ciencias Básicas, Tecnologías e Ingenierías Universidad Nacional Abierta y a Distancia UNAD. Directora Grupo de Investigación GIEPRONAL. 


\title{
The importance of subjectivity for transformative education generation in virtual environments
}

\begin{abstract}
Subjectivities appear as a significant opportunity for education in virtual environments since can be a tool to create real learning. Thus, this paper goes over the concept of subjectivity and the relationships among the cultural, technological and educational contexts. Moreover, is presented a reflection that aims to deepen into the scope and implications of relationships among subjectivities, training processes and virtual education specifically.
\end{abstract}

Key words: subjectivities, virtual environments, education.

Recibido: 25 de abril 2012

Aceptado: 17 de mayo 2012

\section{lntroducción}

Como punto de partida creo necesario plantear dos interrogantes centrales; en primera instancia ¿Cuál es la relación de las subjetividades en el contexto cultural y tecnológico? y de otro lado ¿Cuáles son los alcances e implicaciones de dichas relaciones para la educación y específicamente para la educación virtual?; con el fin de plantear unas posibles respuestas a dichos interrogantes quiero iniciar profundizando en el concepto de subjetividad.

La subjetividad desde la teoría del conocimiento esta relacionada con la existencia de una verdad con una validez limitada; Según, Hessen (1925) «no hay una verdad universal válida», el subjetivismo limita la validez de la verdad al sujeto que conoce y juzga; en esta misma vía, se plantea que existe un subjetivismo individual (juicio válido para el individuo que lo formula) y un subjetivismo general (verdades supraindividuales).

De acuerdo con lo anterior, cuando se habla del subjetivismo se deben tener en cuenta aspectos como las percepciones y los argumentos basados en el punto de vista del individuo, los cuales, se encuentran influidos de manera permanente por intereses particulares y por las circunstancias históricas, políticas y culturales. 
Según Bourdieu, (1992) desde el punto de vista de la filosofía antropológica, la subjetividad se entiende como un proceso de producción de significados que puede analizarse en el nivel individual o en el social. Sin embargo, los significados no solo se generan de alguna manera por los individuos en interacción sino que dentro de ciertos límites espaciales y temporales se vinculan con significados acumulados socialmente que los actores no escogen (Habermas, 1988). Estos significados, no son simplemente compartidos por consenso sino que implican jerarquías sociales y de poder, es decir que existe la posibilidad de la imposición (Foucault, 1976).

Pensar en las subjetividades significa hacerlo sobre las interrelaciones de lo individual y lo colectivo; lo que implica que nos vamos construyendo como seres humanos, en las relaciones con las personas, en la familia, en la escuela y en la comunidad. En este mismo sentido, el contacto con el contexto sociocultural nos proporciona la oportunidad de edificar nuestra subjetividad, nuestra manera de ser, de pensar, de sentir y de actuar.

Husserl (1992), plantea que la subjetividad no esta adentro, ni esta afuera del ser humano, tampoco lo identifica como algo que tengamos que incorporar o que podamos evitar incorporarlo, la subjetividad es trascendental debido a que sitúa todo fenómeno humano en relación con un sistema de significados que de algún modo son los que nos han permitido la convivencia.

De otro lado, el proceso de comunicación se convierte en un medio para que las personas puedan establecer una relación intersubjetiva que conlleve a la construcción de identidades desde lo individual hasta lo social según la comunidad y a la cultura a la cual pertenece. Según Cuche (1996) la importancia actual de la subjetividad nace del rechazo a los determinismos estructuralistas y funcionalistas de épocas pasadas; la subjetividad entonces se ha presentado como una posibilidad de aportar de alguna manera a los sistemas rígidos que han enmarcado algunos contextos sociales y culturales.

Como se puede evidenciar en lo expuesto anteriormente, se hace necesario hacer una relación entre la subjetividad y la cultura; En este sentido, cuando se habla de cultura se entiende como los modos, según Galende (2008), en que todo agrupamiento humano va construyendo significados particulares, para hacer comprensivas y entendibles las relaciones entre unos y otros, y por otra parte de la realización de ciertos valores que permiten jerarquizar los elementos de la vida social.

Ahora bien, según estos planteamientos, la cultura está determinada por ciertas subjetividades que se han venido elaborando con el tiempo, que han sido aceptadas y de alguna manera transmitidas de generación en generación. De igual modo, algunos estudiosos del tema han planteado que existen entre las culturas subjetividades generales que parecen establecer un lineamiento para nuestro comportamiento. 
La importancia de las subjetividades para la generación de una educación transformadora en ambientes virtuales,

Desde esta perspectiva, quisiera ahora traer a este escenario el concepto de tecnología, entendida esta como un tipo de discurso que posibilita la construcción de una técnica que se valida de acuerdo a un contexto sociocultural. Según Quintanilla (2005), las técnicas son entidades culturales de carácter abstracto, que pueden tener distintas realizaciones o aplicaciones y se pueden formular o representar de diferentes formas. Por ejemplo: la técnica de tornear madera utilizando un torno mecánico, el manual de operación del torno sería más o menos el estándar de esa técnica, el cual incluye la descripción de la máquina, el tipo de materiales que se pueden tornear con ella, las operaciones que hay que realizar para utilizarla y seguramente información adicional sobre el arte de tornear con esa máquina.

De acuerdo con los planteamientos que hasta ahora he formulado con respecto a las subjetividades, la cultura, la tecnología y la técnica, quisiera resaltar, cómo el origen de una técnica muy posiblemente tendrá su relación directa con cierto tipo de subjetividades construidas como resultado de situaciones o necesidades vistas desde diferentes perspectivas en forma individual o colectiva; en este sentido, muy seguramente muchos de los desarrollos técnicos y tecnológicos terminaran siendo aceptados, incorporados y transmitidos en un sistema social a través de la cultura.

Las interrelaciones anteriormente mencionadas, nos muestran como nuestra realidad puede ser constantemente cambiada por nosotros mismos cuando ponemos en juego aspectos propios del ser humano. Cabra (2010: 164), en su texto «Video juegos: máquinas del tiempo y mutaciones de la subjetividad» expresa que: «nuestra relación con la tecnología ha abierto umbrales de sentido que han alterado significativamente nuestra percepción y apropiación de la realidad».

Para el caso de las tecnologías de la información y la comunicación (TIC), un tema que resalta especialmente en el ámbito educativo, se ha venido involucrando con ellas una dimensión virtual y de complejas formas de simulación, que de alguna manera pueden poner en riesgo de dispersión la frontera entre lo real y lo imaginado. Según Stone (1996: 96) «los sistemas virtuales implican una comprensión particular del espacio, el tiempo, la proximidad y la agencia».

Aunque hay muchas personas que consideran que la utilización de espacios virtuales para educación no son los más indicados para los propósitos formativos; pienso que, dichos espacios o ambientes virtuales se presentan actualmente como escenarios funcionales para la educación desde diferentes niveles; Las comunidades virtuales representan hoy un gran avance en la renovación de los paradigmas educativos en la medida que posibilitan la formación de redes que permiten atravesar las fronteras de las disciplinas, de las regiones y de las naciones favoreciendo entonces la inter y la transculturalidad. 
En esta misma vía hoy existen muchos que piensan que la interacción entre tecnología, necesidad y contexto social será la que definirá en mayor medida su capacidad de transformación futura. Según lo anterior, la investigación sobre este tipo de tópicos no debe ser abordada desde el reduccionismo tecnológico, sino desde relaciones socio-tecnológicas complejas (Francia, 2005).

Una perspectiva para abordar estas interrelaciones entre la tecnología y las subjetividades en la educación se puede visualizar en el análisis de los procesos comunicativos, tal como lo plantea Cristina Corea en su libro: $L a$ destitución de la comunicación y el agotamiento de la subjetividad pedagógica (2003); para la autora la comunicación es un dispositivo que tenía como elemento clave el código y que para la educación en ambientes virtuales de aprendizaje se transformaría en la información.

El uso de la información en todas sus dimensiones se convierte en un elemento fundamental para el acceso, el análisis, la interpretación, la evaluación y la producción del conocimiento. En la sociedad actual, el proceso de compartir y «transmitir» información se convierte en fuente fundamental de productividad y de poder. En este sentido, las comunidades virtuales con fines educativos se convierten en una nueva forma de construcción de subjetividades y como he venido mencionando a lo largo de este escrito, dichas subjetividades están en la capacidad de influir en la sociedad y en la cultura.

Con lo anterior, he presentado un esbozo muy general de las posibles respuesta a la segunda pregunta formulada: ¿Cuáles son los alcances e implicaciones de las subjetividades y la tecnología para la cultura y la educación en espacios virtuales?; En este sentido y con el fin de mostrar algunos ejemplos puntuales frente a las implicaciones de las subjetividades y la tecnología en la educación, quisiera profundizar un poco en la educación virtual o educación e-learning.

La educación -learning, necesita de un ambiente virtual de aprendizaje (AVA) que permita poner en escena los diferentes elementos que hacen parte de un proceso educativo; en este sentido, dichos elementos han sido objeto de estudio desde hace tiempo atrás, pero quisiera resaltar una problemática que me llama la atención, esta se refiere al desequilibrio que se genera entre la relación y la interacción que se produce entre el docente y el estudiante específicamente.

Según Francia (2005: 4), «existe una sensación de pérdida de control que suelen sentir los profesores virtuales y de esa negación tecnófoga que a muchos docentes inhibe de aproximarse siquiera a estas nuevas tecnologías ya que un entorno virtual puede modificar el ejercicio de poder», este hecho que se convierte en una pequeña parte de la problemática ha sido un factor fundamental para que se presenten resistencias e incredulidades frente a esta modalidad de formación. 
La importancia de las subjetividades para la generación de una educación transformadora en ambientes virtuales,

En mi experiencia como docente de una institución que se apoya fuertemente en el uso de la tecnologías en su proceso formativo, he entendido que los escenarios virtuales también permiten poner en práctica esa expresión que en la actualidad suena un poco a frase de cajón: «debemos construir conocimiento con el otro»; ¿cómo garantizamos que el estudiante pueda construir y gestionar con nosotros el conocimiento?; esta es una pregunta que nos inquieta a muchos docentes en cualquier tipo de ámbito, sin embargo, aun no todos entendemos claramente en la práctica como podemos dar respuesta a dicha pregunta.

El aprovechamiento de las subjetividades e intersubjetividades por los profesores en el proceso formativo, a mi modo de ver, puede convertirse en una gran herramienta para garantizar la construcción de conocimiento; Cuando el sujeto puede hacer interpretaciones de su realidad y además pude identificarla dentro de su proceso de formación de acuerdo a sus intereses, él podría de manera individual y colectiva entender esta realidad y, porque no, transformarla según su criterio.

De acuerdo con Gomez (2005), el internet fue diseñado por humanos y para humanos, según este planteamiento un campus virtual no excluye la relación, la presencia, la comunicación y la significación. Los intereses comunes que permiten dinamizar la interacción entre sujetos son en últimas los que movilizan las acciones que se presentan; en este sentido, un campus virtual se convierte en el escenario donde confluyen e interactúan motivaciones comunes que le permiten al sujeto construir conocimiento con el otro.

El texto y la imagen por ejemplo, aparecen como un apoyo fundamental a los procesos educativos que se desarrollan a través de la virtualidad, en este sentido los docentes deben esforzarse por construir mediaciones que contemplen los medios suficientes y eficientes de tal forma que cuando el estudiante entre en contacto con ellos y a pesar de sus propias subjetividades pueda desarrollar procesos de aprendizaje con el docente aunque no lo conozca; sin embargo, en mi experiencia he entendido que al igual que en un escenario de educación tradicional se hace necesario crear ciertos lazos afectivos con el estudiante, es así, como el docente debe procurar mostrar también su identidad representada no solo con una imagen de su físico (fotografía) si no también desde su experiencia humana (personalidad) representada en la posición que pueda tener desde lo ético, político, social y cultural.

Según lo anterior, se hace necesario avanzar en investigaciones que ayuden a estudiar lo que ocurre socialmente con las relaciones que se originan a nivel virtual, debido a que estas aportan en gran medida en el proceso de aprendizaje dado en un contexto educativo que se apoya en las llamadas tecnologías de la información y la comunicación. Estoy convencida que el producto, desde lo educativo y lo sociocultural, de este tipo de educación es, definitivamente, de un gran impacto positivo en la sociedad, siempre y cuando se lleve de la forma adecuada. 
Como apoyo a lo planteado hasta el momento, quisiera referir varios elementos tratados por Margarita Victoria Gomez en su libro Educación en red, en el que se refiere al sujeto pedagógico en la red, el cual, según ella, se piensa en el juego del múltiple movimiento entre el «yo individual y el yo social»; «el acaso» planteado por un estudiante frente a una situación determinada (considerando como acaso un nuevo hecho a realizar), para la educación tradicional era un error y por consiguiente estaba descartado o visto con prejuicio, pero para la educación virtual «el acaso» puede ser considerado como una oportunidad de actualización, en el sentido que este permite integrar en el escenario de formación pensamientos diferentes y singulares, los cuales enriquecen en gran medida tanto a docentes como estudiantes.

Estos participantes, pueden entonces reconocerse como eternos aprendices, en la medida que se relacionan con ellos mismos y con los otros, de esta manera se dan las condiciones para que puedan reflexionar sobre ellos frente a su quehacer. Lo anterior se apoya en cuatro aspectos fundamentales: la construcción colectiva, la comunicación, el diálogo y el encuentro entre sujetos; en este sentido quiero retomar como apoyo a mi discurso las siguientes citas de Margarita Gomez que me parecen reveladoras:

1. «La construcción colectiva emergente en la multiplicidad de los intercambios, puede sustentar un trabajo pedagógico que permita desabrochar una pedagogía nómada, viajera, itinerante de la virtualidad, al dar subsidios para la constitución de subjetividades productivas, deseantes y revolucionarias, en una red simbólica por excelencia, preocupada más con la conexión y la creatividad que con el simple contacto para la acumulación de conocimientos» (Gomez, 2005).

2. «La comunicación, el diálogo y el encuentro entre sujetos, que llegan con sus preguntas más que con sus certezas, van delimitando permanentemente nuevos espacios de subjetivación que generan una cultura particular para que la educación sea realizada en ese sentido de posibilidad» (Gomez, 2005).

Pero entonces, ¿cómo generar y hacer uso de subjetividades en un escenario de educación virtual?; para dar respuesta a este interrogante quiero que pasemos a una dimensión que parece ser de tipo instrumental pero que si lo miramos con detenimiento iría mas allá. En cualquier proceso formativo se hace necesario establecer diferentes tipos de escenarios que faciliten la interacción entre el docente, el estudiante y el conocimiento, los escenarios virtuales que directamente responden a unas intencionalidades de tipo pedagógico y didáctico están apoyados por medios que se fundamentan entre otras cosas en la posibilidad de establecer herramientas de comunicación sincrónicas (en el mismo tiempo) o asincrónicas (en distinto tiempo) de acuerdo a la plataforma tecnológica en la cual se trabaja. 
La importancia de las subjetividades para la generación de una educación transformadora en ambientes virtuales,

Las herramientas de comunicación mencionadas deben estar dirigidas a propiciar aprendizajes de tipo colaborativo y autónomo, los cuales, interrelacionados, facilitaran la construcción de conocimiento. La forma en que se dinamizan los procesos comunicativos, en un aula virtual, es fundamental para la labor formativa, y en este sentido la velocidad en los cambios tecnológicos ha generado herramientas cada vez más eficientes para abordar este tipo de procesos.

Según Castañeda, (2007: 2) «La utilización de herramientas de comunicación telemática que permiten el intercambio textual, por voz y/o audiovisual, es un fenómeno creciente en nuestros tiempos y que -junto con el intercambio textual a través de mensajes cortos y desde los teléfonos celulares, los conocidos SMSha cambiado radicalmente la forma en la que las personas se relacionan en el día a día». Para la educación este hecho ha permitido fortalecer su dinámica en el sentido en que se ha abierto un gran abanico de posibilidades de interacción. Hoy encontramos otros medios que permiten acercar las relaciones entre estudiantes y docentes; entre estos se encuentran por ejemplo: los mundos virtuales y la realidad aumentada. Los primeros permiten estar en un ambiente inmersivo y dinámico, facilitando la ejecución de actividades grupales de individuos en tiempo real. Estos escenarios posibilitan la generación de nuevas subjetividades que seguramente enriquecerán el aprendizaje en el sujeto de forma individual y colectiva.

Sin embargo, cabe mencionar que este tipo de herramientas por si solas no desarrollaran procesos de aprendizajes, es importante resaltar que por ningún motivo la tecnología debe estar por encima de su uso pedagógico; de acuerdo a lo anterior, la ventaja de los mundos virtuales para la educación deber estar en el cómo usarlos según las intencionalidades formativas propuestas por el docente. De acuerdo con lo planteado por diferentes personas dedicadas a desarrollar este tipo de aplicaciones para la educación: «los mundos virtuales deben ser herramientas que le permitan al estudiante enfrentarse a situaciones en donde tenga que aplicar nuevos conocimientos enfrentándose a su vez nuevos retos». (3Dsoft Blog, 2011)

En el segundo caso, tenemos la realidad aumentada, la cual se manifiesta como interacción que trata de reducir el intercambio con el ordenador utilizando la información del entorno como una entrada implícita. Con este estilo el usuario será capaz de interaccionar con el mundo real, aumentado por la información sintética del ordenador. Se trata entonces de una nueva tecnología que aumenta o mejora la visión que el usuario tiene del mundo real con información adicional sintetizada mediante un modelo computarizado. Los usuarios pueden trabajar y examinar objetos 3D reales mientras reciben información adicional sobre estos objetos o sobre la tarea que se está realizando. (Alonso, et. al, 2000)

Estos dos ejemplos de desarrollos tecnológicos aplicados a la educación, nos mueven a ir más allá de lo instrumental, del reduccionismo al cual hemos llegado 
como resultado del desconocimiento de los diferentes elementos y relaciones que intervienen en los procesos educativos. En este sentido, es importante hacer entender a los docentes que de nada sirve utilizar los más avanzados dispositivos tecnológicos si se siguen repitiendo esquemas trasmisionistas y conductistas que se vienen manifestando desde hace muchos años en la educación tradicional.

Igualmente, quisiera dejar en evidencia la necesidad de aprovechar y dimensionar la construcción de subjetividades en los procesos de formación que se generará en un escenario educativo de tipo virtual, lo cual se puede entender como una herramienta que conlleve a una apuesta de educación pertinente con el contexto y que esté en la capacidad de transformación de la sociedad.

\section{Referencias Bibliográficas}

Alonso, N., Balaguer, A; Bori, S; Ferré, G; Junyent, E; , Lafuente, A., López, J. B., Lorés, J., Muñoz, D., Sendín, M., Tartera, E. (2001). "Análisis de escenarios de futuro en realidad aumentada. Aplicación al yacimiento arqueológico de Els Vilars". En: Interacción'2001, 2 Congreso Internacional de Interacción Persona-Ordenador, Salamanca, Mayo 2001.http://griho2.udl.cat/publicacions/2001/Interaccion_2001_Realidad_Aumentada.pdf [consultado: 25.02.2011].

Bourdieu, P. 1992. The Logics of Practice. London: Polity Press.

Cabra, N. 2010. «Videojuegos: máquinas del tiempo y mutaciones de la subjetividad». En: Signo y Pensamiento. XXIX:57:162-177.

Castañeda Quintero, L. (2007) “Herramientas Sincrónicas Y CuasiSincrónicas Para La Comunicación Educativa”. En PRENDES ESPINOSA, M. P. Herramientas Telemáticas Para La Enseñanza Universitaria En El Marco Del Espacio Europeo De Educación Superior. Grupo de Investigación de Tecnología Educativa. Universidad de Murcia. pp 1-18 CD - ROM. ISBN: 978-84-611-7947-3

Corea, C.. 2003. La destitución de la Comunicación y el agotamiento de la subjetividad pedagógica. Buenos Aires: Documento de FLACSO.

Cuche, D. 1996. La noción de cultura en las ciencias sociales. Buenos Aires: Nueva Visión.

Foucault, M. 1976. Vigilar y Castigar. México: Siglo XXI.

Francia, A. L. 2005. «Desde la subjetividad pedagógica tradicional a la virtual». Revista Iberoamericana de Educación. 35 (5):1-5 


\section{Milena Alcócer Tocora}

La importancia de las subjetividades para la generación de una educación transformadora en ambientes virtuales, artículo de reflexión

Galende, E. 2008. El impacto de la cultura en la subjetividad de las personas. Universidad Nacional del Rosario. En encuentro AVCD, página web: http://www.avcd-argentina. org/pdfponencias/galende.pdf.

Gomez, M.V. 2005. Educación en red: una visión emancipadora para la formación. Guadalajara: Universidad de Guadalajara.

Habermas, J. 1988. La Lógica de las Ciencias Sociales. Madrid: Tecnos.

Hessen, J. 1925. Teoría del Conocimiento. Bogotá: Ediciones Universales.

Husserl, E. 1992. La crisis de las ideas europeas y la fenomenología trascendental, Barcelona: Editorial Crítica.

Quintanilla, M. 2005. Tecnología: un enfoque filosófico y otros ensayos de la filosofía de la tecnología. México: F.C.E.

Stone, A. R. 1996. The War of Desire and Technology at the Close of the Mechanical Age, Massachusetts: Massachusetts Institute of Technology.

3Dsoft Blog. 2011. ¿Por qué usar mundos virtuales en la educación? Página Web:http://o3dsoft.com/blog/es/2011/04/por-que-usar-mundos-virtuales-en-la educacion/?wpmp_switcher=mobile [consultado: 02.08.2012]. 